\title{
High Speed Novel Design of Sram for Highly Reliable Applications
}

\author{
D.naresh, s.butchi babu, S. Baba fariddin, S. Ravindra
}

\begin{abstract}
Modern ICs are enormously complicated due to decrease in device size and increase in chip density involving several millions of transistors per chip. The rules for manufactured leads to a tremendous increase in complexity due to the amount of power dissipation are increased. In this paper, the design of novel SRAM is implemented for the highly reliable applications. For high-speed memory applications such as cache, a SRAM is often used. Power consumption is the key parameter for an SRAM memory design (SRAM). New tag generation system designed for integrity checking of SRAM. A single read operation to a crossbar SRAM that can be used for integrity checking. Reliability of the system is measured for varying conditions of device parameters, operating temperatures, load resistances, and read voltage.
\end{abstract}

KEY WORDS: SRAM (static random access memory), DRAM (dynamic random access memory), RAM (random access memory).

\section{INTRODUCTION}

Quick developing business sector of convenient electronic gadgets (e.g., phone, individual computerized right hand, MP3 player, advanced camera, and so on.) has made a developing interest for recollections. Recollections are a sort of non-unstable memory dependent on floating rate transistors putting away and evaluating charge on the FG electrically. The in-field programmability and low power utilization gain streak experiences widely utilized for compact gadgets. Different charge instruments, cell structures, and cluster designs have been created previously. Recollections likewise can be inserted in a framework chip to permit programming refreshes [1]. Embedded memory has demonstrated a rapid growth over the past few years and has emerged as one of the essentialness misshape in the midst of forming is more than the imperativeness adversity in the midst of examining in common SRAM since there is full swing of voltage in bit lines while the bit line voltage swing is less in the midst of scrutinizing. It was understood that the essentialness set away in the bit lines of the customary SRAM is lost to ground in each make task in the midst of ' 1 ' to ' $O$ ' advancement and this is the guideline wellspring of imperativeness hardship [2-3].

The power scattered in bit lines talks about the total special power usage in the midst of a make task.

Revised Manuscript Received on December 13, 2019.

Correspondence Author

${ }^{1}$ D.Naresh, Assistant Professor, Dept. of E.C.E, Lakireddy Balireddy College of Engineering, Mylavavaram, A.P

${ }^{2}$ S.Butchi Babu, Assistant Professor, Dept. of E.C.E, Vikas College of Engineering and Technology, Nunna, Vijayawada, A.P

${ }^{3}$ S. Baba Fariddin, Assistant Professor, Dept. of E.C.E, St.Mary's Women's Engineering College, Guntur, A.P and pursuing Ph.D at Dr. A.P.J Abdul Kalam University, Indore, M.P.

${ }^{4}$ S. Ravindra, Assistant Professor, Dept. of E.C.E, St.Mary's Women's Engineering College, Guntur, A.P

The power use by bit lines in the midst of forming is comparing to the bit line capacitance, square of the bit line voltage and the repeat of making. Unwavering quality has been viewed as the essential issue for glimmer recollections. They are generally tried for unsettling influence issues, including read-bother issue, program irritate flaw, eradicate aggravate deficiency, and so on. Customarily, a test method for the aggravate deficiencies is typically specially appointed. What's more, traditional March tests which are created in the past to test RAMs are not proper for testing streak recollections. In contrast to a RAM, there are three distinct activities for a memory read, program, and eradicate [4]. The read task per uses the information put away in a memory word; the program activity charges the cells of a word, while the glimmer delete task releases every one of the cells in an area (a segment eradicate) or the whole chip (a chip-eradicate). Most glimmer recollections can complete an irregular read and arbitrary program (compose 0) however can't do arbitrary delete (compose 1). Rather, they do streak delete, i.e., part eradicate or chip eradicate. [5].

In most security applications, many security schemes including encryption, integrity checking, and authentication are adapted to protect sensitive data. Due to their vital roles, the secret keys must be stored with improved security, especially resisting physical attacks, which include fully invasive attacks, side-channel attacks, malicious writing or fault induction, etc. Current keys storage methods can be distinguished between off-chip and on-chip storage.

The off-chip storage generally uses external non-volatile memory (NVM) such as EEPROM/Flash to store keys, but is vulnerable to data interception attack cross chip boundary. The emerging resistive random access memory (RRAM) has attracted research enthusiasm from materials, devices and chips. Until now, this research mainly focused on improving performance including high to low resistance window, data retention, endurance, etc, and the fabricated chips mainly addressed the circuit and architecture issues for high density standalone applications [6-8]. This paper proposed a SRAM chip for keys storage with physical security features of resisting fully invasive attacks, side-channel attacks, malicious writes and data interception, etc. The RRAM chip was implemented on the same chip with a demonstrative information security platform in $0.13 \mathrm{um}$ standard logic technology, as keys storage. Experiments that emulated reverse engineering, encryption/decryption and side-channel attacks provide the security features. Compared with the anti fuse counterpart, the proposed RRAM chip has smaller area, lower write voltage and power, faster read speed, and more security features, which make it more suitable for keys storage. 


\section{High Speed Novel Design of Sram for Highly Reliable Applications}

\section{CHARACTERISTICS OF PROPOSED SYSTEM}

\section{a. Operating Voltage}

It is outstanding that a high working voltage is a lethal defect in commonsense application. This is on the grounds that a high working voltage implies high power utilization. Also, unwavering quality can be an issue with a high working voltage. To pick up favorable position over flash memory, which suffers from high working voltages, both the programming and deleting voltages for RRAM gadgets ought to be just a couple of volts.

\section{b. Operating Speed}

Working rate is characterized as the most limited time for programming or deleting a gadget cell. Lee et al. had given an account of the bipolar resistive exchanging attributes of a RRAM gadget. The exchanging velocity of this gadget is as quick as $5 \mathrm{~ns}$, the quickest speed yet announced. Right now, the best working rate of RRAM gadgets is somewhere in the range of 5 and $100 \mathrm{~ns}$.

\section{c. Resistance Ratio}

Opposition proportion, the proportion of the obstruction at HRS to the opposition at LRS, assumes a significant job in RRAM applications since it legitimately impacts the exactnesses of programming and deleting. When all is said in done, an obstruction proportion more noteworthy than 10 is required to recognize the two opposition states in circuit plan. In some RRAM gadgets, the obstruction proportion can be as high as six or seven requests of extent. Such an immense obstruction proportion recommends the likelihood of accomplishing high-thickness memory by methods for multibit or staggered stockpiling.

\section{d. Endurance}

A RRAM gadget can be exchanged among HRS and LRS every now and again, yet every task can present lasting harm, regularly alluded to as debasement. Perseverance, likewise called electric weakness, is the quantity of set/reset cycles that can be suffered before HRS and LRS are never again discernable. To pick up preference over Flash memory, which demonstrates a most extreme number of compose cycles somewhere in the range of 103 and 107 , relying upon the sort, RRAM ought to give at any rate a similar continuance, ideally a superior one.

\section{e. Retention Time}

Maintenance time, the time allotment a memory cell will remain in one state in the wake of programming or eradicating, demonstrates the characteristic capacity of a memory cell to hold its substance. Most monetarily accessible items are ensured to hold data for something like 10 years, either with the power on or off.

\section{f. Multilevel Storage}

Staggered stockpiling alludes to the capacity of a memory cell to display reproducible resistive exchanging between numerous opposition states and, thusly, to store different qualities. Staggered stockpiling can upgrade stockpiling thickness, yet requires a sufficiently substantial obstruction proportion between each state to empower an outside circuit to recognize states. Staggered stockpiling can be accomplished by controlling either consistence present or working voltage. In our past work, a WO3 based RRAM gadget with $\mathrm{Cu} / \mathrm{WO} 3 / \mathrm{Pt}$ structure was shown to have staggered capacity ability. Amid the set procedure, by utilizing distinctive consistence flows, no less than four obstruction states can be tended to, with each state relating to a dimension of memory.

\section{g. Device Yield}

Non stoichiometric oxide gadgets for the most part have a lethal defect of low gadget yield because of the wildness of the oxygen fixation. To tastefully tackle the issue of low gadget yield for non stoichiometric oxides, $\mathrm{Wu}$ et al. used stoichiometric $\mathrm{ZrO}_{2}$ rather than non stoichiometric $\mathrm{ZrO}_{2}$ for nonvolatile RRAM gadgets. Likewise, different strategies have been proposed to improve gadget yield, for example, utilizing an appropriate terminal material, using doped metal oxide, and purposefully presenting metal nano precious stones in oxide.

\section{RELATED WORK}

These days Static Random Access Memory (SRAM) has turned into an irreplaceable part of advanced frameworks, which can be utilized as an independent item or installed recollections in a System on Chip (SoC) item. The quantity of SRAM centers in a SoC is expanding drastically in light of the structure necessity of encouraging various applications. This prompts ever-higher thickness and ever beyond words. Then again, innovation scaling prompts smaller element sizes while empowering countless to be manufactured into a solitary chip. In any case, such innovation scaling additionally prompts higher danger of obscure deformities that arbitrarily happen in such an immense number of memory cells.

Along these lines, fault diagnosis and troubleshooting of SRAM are confounded and require effective test calculations. Advancements in embedded memory innovation have made substantial Dynamic Random Access Memories (DRAMs) and Static Random Access Memories (SRAMs) typical in the present System on Chips (SoCs.) Tradeoffs among vast and little recollections have made all sizes handy, empowering SoCs to look like board-level frameworks like never before. Vast implanted recollections give a SoC various advantages, for example, enhanced transfer speed and impressive execution that must be accomplished using installed advances. The possibility and achievement of including installed DRAM as well as vast SRAM obstructs in a SoC depends predominantly on manufacturability. By and large in battery fueled applications like took care of keen gadgets and embed restorative gadgets. The low power tasks are the serious issues related with framework on chip (SoC). Later this low power framework on chips is acknowledged with the low power static arbitrary access memory. In this SRAM impacts the all out intensity of SoC and possesses the extensive part of territory of SoC. Due to this there is decrease in power and low working voltage impacts the varieties in edge voltage. To acquire high thickness reconciliation, in SRAM cell little transistors are utilized. Going to the SRAM, it comprises of both read and composes soundness in low voltage locale. The primary favorable position of including decoupled read port is that it dispenses with the exchange off between read dependability and composes soundness. There are delicate mistakes happened in the SRAM cell, to Delimitate these blunders we utilize the bit interleaving SRAM engineering. 
Recollections additionally can be implanted in a framework chip to permit programming refreshes. Inserted memory has exhibited a quick development in the course of recent years and has risen as one of the quickest developing parts in the implanted gadget advertises. The developing interest of compact battery worked frameworks has made vitality effective processors a need. Decrease of the supply voltage of the SRAM cells is known to adverse affect the information steadiness of the SRAM cells. A SRAM cell can store one piece of information. A SRAM cell contains two consecutive associated inverters shaping a hook and two access transistors. Access transistors serve for read and compose access to the cell. A SRAM cell can hold the information inconclusively as long as it is controlled.

The information of a SRAM cell can be set to any paired esteem paying little respect to its unique information. Static information soundness of the SRAM cell has been a conspicuous subject in the SRAM cell plan. This is on the grounds that it inspects the SRAM cell for its capacity to play out its principle task; to hold the information. The notion of static data stability is the foundation of the realization of binary computing using electrical devices such as BJT and MOS transistors for decades. Basically, this notion links the physical voltage levels at the input and the output of a gate (e.g., an inverter) to the Boolean logic states. A gate can offer a logic operation if for any arbitrary static input, the static output voltage of an infinitely long chain of the gate converges to one of the three unique voltage levels associated with the gate.

\section{NOVEL SRAM DESIGN}

Figure (1) shows the block diagram of Novel SRAM design. In this mainly, word line decoder, shift register, read line decoder, control unit and error correction code is used. The entire operation is controlled by the control unit. SRAM array will save the information in the form of bits by using array formation. Cell checking will check the bits which are coming from the decoder unit. Read line decoder will decode the data which is already stored and error correction code will check if there are any errors occurred or not.

SRAM or Static Random Access Memory is a type of semiconductor memory broadly utilized in gadgets, microchip and general registering applications. This type of semiconductor memory picks up its name from the way that information is held in there in a static design, and shouldn't be progressively refreshed as on account of DRAM memory.

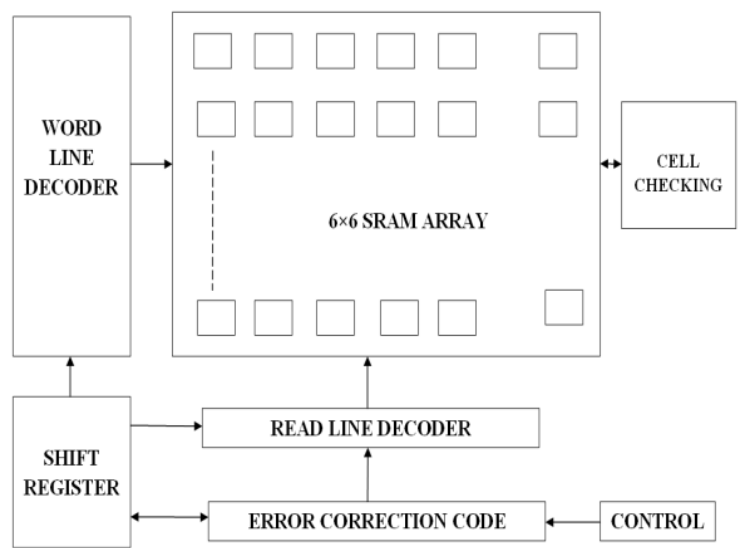

Fig. 1: BLOCK DIAGRAM OF NOVEL SRAM

While the information in the SRAM memory shouldn't be invigorated progressively, it is as yet unpredictable, implying that when the power is expelled from the memory gadget, the information isn't held, and will vanish. The circuit for an individual SRAM memory cell contains regularly four transistors arranged as two cross coupled inverters. In this arrangement the circuit has two stable states, and these liken to the intelligent "0" and "1" states. Access to the SRAM memory cell is empowered by the Word Line. This controls the two access control transistors which control whether the cell ought to be associated with the bit lines. These two lines are utilized to move information for both read and compose activities.

The word line decoder is utilized to choose specific segment in the memory exhibit. The run of the mill section decoder in which the yields of 2 to 4 decoder are utilized to empower pass transistors. The Boolean capacity of the decoder is practically identical to $n$-information AND rationale entryways, where the broad fan-in AND task is completed in a different leveled structure. The arrangement of these decoders note worthily affects the speed and power dispersal of the memory. The main dimension is the pre decoder where two gatherings of location information sources and their supplements are first decoded to start one of the pre-decoder yield wires independently to get the mostly decoded yields. The pre-decoder yields are united at the accompanying dimension to empower the word line. The decoder postponement includes word line wire delay, interconnect deferral of pre-decoder and door delays in the basic way. The below figure (2) shows the schematic view of novel SRAM.

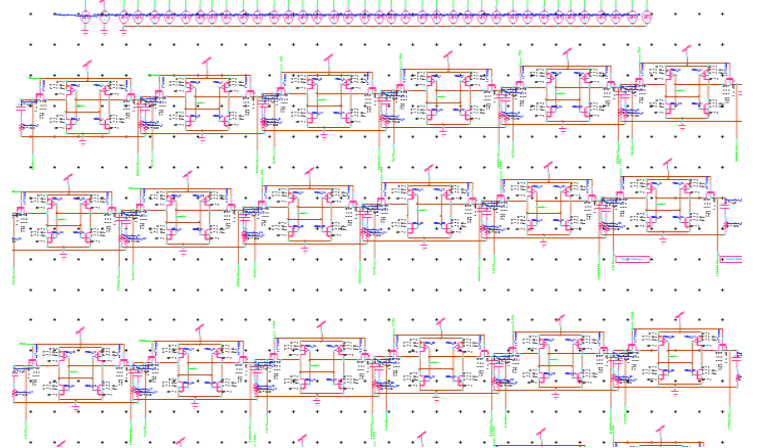

Fig. 2: SCHEMATIC VIEW OF NOVEL SRAM

V. RESULTS

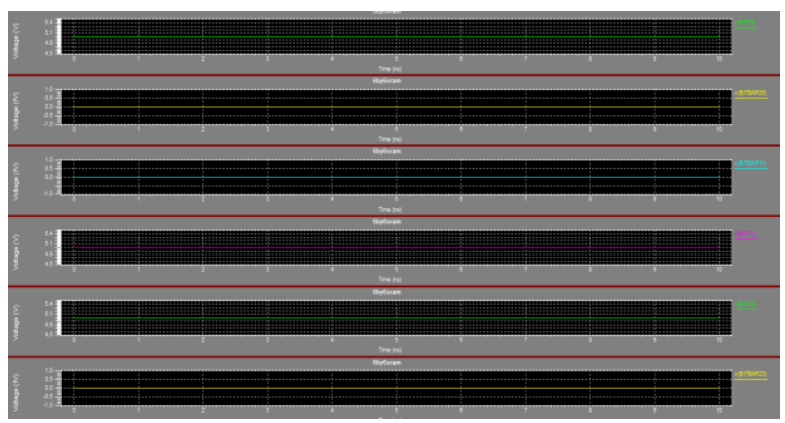

Fig. 3: OUTPUT WAVEFORM OF NOVEL SRAM DESIGN 


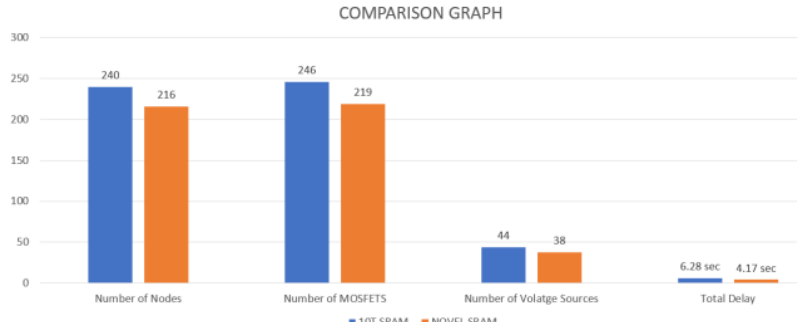

Fig. 4: COMPARISON GRAPH

Table. 1: COMPARISON TABLE

\begin{tabular}{|c|c|c|}
\hline PARAMETERS & 10T SRAM & $\begin{array}{c}\text { NOVEL } \\
\text { SRAM }\end{array}$ \\
\hline $\begin{array}{c}\text { NUMBER OF } \\
\text { NODES }\end{array}$ & 240 & 216 \\
\hline $\begin{array}{c}\text { NUMBER OF } \\
\text { MOSFETS }\end{array}$ & 246 & 219 \\
\hline $\begin{array}{c}\text { NUMBER OF } \\
\text { VOLTAGE } \\
\text { SOURCES }\end{array}$ & 44 & 38 \\
\hline $\begin{array}{c}\text { TOTAL } \\
\text { DELAY }\end{array}$ & $6.28 \mathrm{sec}$ & $4.17 \mathrm{sec}$ \\
\hline
\end{tabular}

\section{CONCLUSION}

This algorithm is quite fast and can provide excellent solutions in short run-times. Experimental results indicate that this flow can save the designer many days of work by offering good architectures which are complete in terms of logical and physical attributes. The design of SRAM memory cells to be a challenging and valuable learning experience. The static RAM is very widely used in CMOS systems. It is strongly believed that the proposed system can be widely used for the embedded memory testing especially under the SoC design environment due to the superior flexibility and extendibility in applying different combination of memory test algorithms.

\section{REFERENCES}

1. Himanshu Banga, Dheeraj Agarwal, "Single Bit -Line 10T SRAM cell for Low power and High SNM", Proceeding International conference on Recent Innovations is Signal Processing and Embedded Systems (RISE -2017) 27-29 October,2017.

2. Ching-Wei, Ming-Hung, "A Configurable 2-in-1 SRAM Compiler with Constant-Negative-Level Write Driver for Low Vmin in 16nm Fin-FET CMOS", 978-1-4799-4089-9/14/\$31.00 @ 2014 IEEE.

3. Akshay Bhaskar, "Design and Analysis of Low Power SRAM Cells", International Conference on Innovations in Power and Advanced Computing Technologies [i-PACT2017].

4. Ik-Joon Chang, "Priority based SRAM Design", 978-1-4799-4089-9/14/\$31.00 @ 2013 IEEE.

5. Chinmay Sharma, Varun Chhabra , Balwinder Singh, "Design of SRAM array using Reversible logic for an efficient SoC design", Proceedings of the International Conference on Inventive Computing and Informatics (ICICI 2017) IEEE Xplore Compliant - Part Number: CFP17L34-ART, ISBN: 978-1-5386-4031-9.

6. Rouwaida Kanj, Rajiv Joshi, Sani Nassif, "Mixture Importance Sampling and Its Application to the Analysis of SRAM Designs in the Presence of Rare Failure Events", DAC 2006, July 24-28,2006, San Francisco, California, USA.

7. Huifang Qin, Animesh Kumar, Prakash Ishwar, "Error-Tolerant SRAM Design for Ultra-Low Power Standby Operation", 0-7695-3117-2/08 \$25.00 () 2008 IEEE.

8. S. Kiamehr, F. Firouzi, and M. B. Tahoori, "A layout-aware X-filling approach for dynamic power supply noise reduction in at-speed scan testing," in Proc. IEEE Eur. Test Symp., May 2013, pp. 1-6.

9. M. Nourani, M. Tehranipoor, and N. Ahmed, "Low-transition test pattern generation for BIST-based applications," IEEE Trans. Comput., vol. 57, no. 3, pp. 303-315, Mar. 2008.

10. Wan Hassan, W.Z, Othman, M., Suparjo, B.S., "A Realistic March-12N test And Diagnosis Algorithm For SRAM Memories" IEEE International Conference on Semiconductor Electronics, pp. 919 - 923, 2 July 2007

\section{AUTHOR PROFILE}
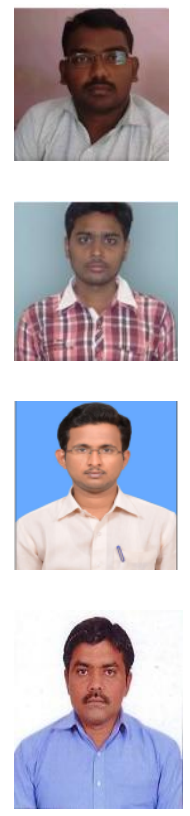

D Naresh working at Lakireddy Balireddy College of Engineering, Mylavavaram. He has 6 years of Experience.

S BUTCHI BABU working as assistant professor at Vikas college of engineering and technology, Nunna, VIJAYAWADA rural. He has 5 years of teaching experience.

S BABA FARIDDIN Working as Assistant Professor at St.Mary's Women's Engineering College, Guntur. He has 6 years of teaching experience and pursuing $\mathrm{Ph} . \mathrm{D}$ at Dr. A.P.J Abdul Kalam University, Indore. His area if interest is VLSI design.

S. RAVINDRA Working as Assistant Professor at St.Mary's Women's Engineering College, Guntur. He has 6 years of teaching experience. His area of interest is VLSI design. 